\title{
4
}

\section{The Treatment of Vascular Dementia in Acupuncture Based on Syndromes Differentiation}

\author{
Liangdeng Zhang1,2, Jie Wang1, Kuiwu Yao ${ }^{1}$ and Ji Zhang ${ }^{2}$ \\ ${ }^{1}$ Guang'anmen Hospital, China Academy of Chinese Medical Sciences, Beijing \\ ${ }^{2}$ School of Acupuncture and Moxibustion, Beijing University of Chinese Medicine, Beijing \\ China
}

\section{Introduction}

Vascular dementia $(\mathrm{VaD})$ is a progressive neurodegenerative syndrome that encompasses a wide spectrum of cognitive disorders caused by cerebrovascular diseases (Pennisi et al., 2011; Selnes \& Vinters, 2006). VaD and Alzheimers disease (AD) are the most common dementia syndromes in the elderly (Matioli \& Caramelli, 2010). However, only a small percentage of elderly patients have 'pure $\mathrm{AD}^{\prime}$ or 'pure $\mathrm{VaD}^{\prime}$ (Fotuhi et al., 2009), both of which are caused by mixed aetiology and pathophysiology. Clinical diagnostic criteria of VaD show moderate sensitivity (50-70\%) and variable specificity (64-98\%) (Jellinger, 2008). The frequency of specific neuropathologic features of vascular cognitive impairment depends largely on study exclusion criteria (Selnes \& Vinters, 2006). Although the use of voxel-based multivariate analysis in 18FDG PET extracted a metabolic pattern could differentiate $\mathrm{VaD}$ and $\mathrm{AD}$ efficiently (Kerrouche et al., 2006). VaD is in silent epidemic now as global ageing population developing, while its prevalence ranges from 4.5 to $39 \%$ with standardized incidence rates between 0.42 and 2.68 per 100,000 world people (Jellinger, 2008), caused by cerebrovascular disorders and vascular risk factors, such as stroke, generalized cerebral atherosclerosis, hypertension, hypercholesterolaemia, especially progressive cerebrovascular atherosclerosis and consecutively stroke in asian and western countries (Konno et al., 1997). There are many subtypes of $\mathrm{VaD}$, such as cortical VaD, subcortical $\mathrm{VaD}$ and cortical-subcortical $\mathrm{VaD}$. The mechanisms that lead to $\mathrm{VaD}$ are controversial and remain to be elucidated (Schuff et al., 2009). Gene-environment interactions are central to human disease (Traynor \& Singleton, 2010), as well as VaD. Virusgene interactions also should not be ignored (Dobson et al., 2003). Telomere length may be an independent predictor for the risk of $\mathrm{VaD}$ (Zglinicki et al., 2000).

The name of $\mathrm{VaD}$ is chidai in traditional Chinese medicine (TCM). The earliest description of chidai ascends to Pre-Qin Dynasty in China, such as the book Zuozhuan said 'Chidai is the meaning of not clever.' Huangdi's Internal Classics recorded the classic understanding of VaD correlated with age in TCM, as it said 'Heart qi begins weakness at sixty years old, the elderly are fond of being anxious and depressed, while blood and $q i$ are apt to delinguency, which lead to be keen on decubitus or sleep; Lung qi begins weakness at eighty years old, the soul of the elderly are dispersion, which lead to be often mistake on communication.' 
Huangdi's Internal Classics also said 'Head is the house of intelligence... Heart is the viscus likened to the monarch in the body which produces the intelligence... Heart is as a major regulator of five zang-organs and six fu-organs and is also the house of spirit... Heart stores the spirit, lung stores the soul, liver stores the mentality, spleen stores the will, and kidney controls the aspiration.' Compendium of Materia Medica proposed definitely that 'Brain is the house of cerebral spirit'. As we know, both the patterns of holism and treatment with syndromes differentiation are the special characteristics of TCM. So the brain is the location of disease of $\mathrm{VaD}$, as well as the heart in TCM. Besides, the disorders of any of five zangorgans and six fu-organs could lead to cognition impairment and VaD. For example, heart stores the spirit and kidney is the origin of primordial qi and yinyang, both the two viscera could be as assistance for the brain to manage spirit. As Syndromes Differetiation Record said 'The integration of heart and kidneys produce wisdom, while the failure of which lead to wisdom loss'.

\section{The primary etiology and pathogenesis of $\mathrm{VaD}$ in TCM}

The characteristics of disease of $\mathrm{VaD}$ in $\mathrm{TCM}$ are asthenia in origin and sthenia in superficiality, as well as deficiency syndrome mingling with excess syndrome. There are five highlights on primary etiology and pathogenesis of $\mathrm{VaD}$ in TCM while integrating our decades of years for TCM clinical practice. The five primary points on understanding VaD in TCM should be integrated with western medicine to guide for clinical practice (Zhang, 2010).

\subsection{Ageing and asthenia of healthy energy accompanying with concurrent diseases of zang-organs and fu-organs}

The functions of zang-organs and fu-organs are degenerative in the elderly as age growing. Huangdi's Internal Classics said 'The male's kidney qi begins weakness at forty years old, while liver $q i$ begins weakness at fifty-six'. On the one hand, yin qi is degenerative with aging, both the essence and blood are deficient, as well as marrow-reservoir, which lead to loss of brain nourish and dysfunction of the mind and psychoactivity. On the other hand, the essence and blood of liver and kidneys are insufficient, hyperactivity of fire caused by deficiency of yin that leads to endogenous deficient wind, which induce activities discomforts. Besides, heart and spleen are deficient gradually with aging, heart deficiency leads to dysfunction of the mind while spleen deficiency causes insufficiency of $q i$ and blood, which lead to deficiency of essence and marrow and dull of the mind.

\subsection{Autolesion of zang-organs and fu-organs caused by seven emotions disorder} Seven emotions include the joy, rage, anxiety, contemplation, sorrow, fear and surprise in TCM. Huangdi's Internal Classics said 'rage drives $q i$ upward, overjoy slackens $q i$, excessive sorrow consumes $q i$, fear disorders $q i$, over-strain consume essence, anxiety causes $q i$ stagnation.' The disorder of seven emotions could lead to dysfunction of $q i$ and blood, lack nourish of heart mind and brain sea, which turn out to be abnormal mind and cognition impairment and dementia.

\subsection{Five excessive emotional activities and incoordination of seven emotions}

Five emotional activities correlate with functions of five zang-organs closely, including joy, rage, anxiety, contemplation, and fear. Huangdi's Internal Classics said 'heart associates with 
joy, liver connects with rage, spleen associates with contemplation, lungs govern anxiety, and the emotion of the kidneys is fear.' Excess of liver qi causes rage, pathogenic fire derives from stagnation of liver $q i$, aberration of heart mind, hyperactivity of both fire of heart and liver, all of which lead to be insufficiency of essence and blood and marrow sea and dysfunction of the mind, then, turn out cognition impairment and dementia.

\subsection{Turbid phlegm blocks the clear orifices and clear yang fails to distribution}

The brain is the house of clear yang and upper $q i$ in TCM. If the upper qi was not enough, the brain sea would be deficient. On one hand, internal stagnation of phlegm would be occurrence caused by kidneys deficiency, or excessive contemplation injuring spleen, then, transformation failure of stomach and intestine and metabolic disorder of body fluid. On the other hand, long addiction to fatty and sweet food and smoking and alcohol abuse lead to stagnation of qi due to phlegm and damp. All of which cause turbid phlegm to block the willing, yet, the brain would be deficient and dysfunction of the mind, thus, lead to dementia.

\subsection{Blood stasis obstructs the collaterals and deficiency of marrow-reservoir}

Blood is one of the most treasurable objects of human body as well as $q i$, both of which enriched in the brain sea are the source of wisdom. 'If inside blood stasis occurred, the patient would turn out morbid forgetfulness and madness' said in Systematic Compilation of Internal Classic. Blood stasis or deficiency in brain would lead to abnormal mind. 'Demented syndrome caused by $q i$ and blood stagnation' also said in Errors in Medicine Corrected. Cognitive impairment is caused by qi stagnation and blood stasis in meridians and collaterals, especially after stroke as aging.

\section{Treatment of $\mathrm{VaD}$ in acupuncture based on syndromes differentiation}

The treatment of $\mathrm{VaD}$ in acupuncture has thousands of year origin in TCM. Yulong Classics on Acupuncture and Moxibustion said 'Shenmen (HT 7) could treat dementia alone.' Classified Classics on Acupuncture and Moxibustion said 'Neiguan (PC 6) could regulate the impaired cognition.' Compendium of Acupuncture and Moxibustion also said 'Baihui (DU 20) could regulate wind syndrome of head and stroke, pavor and morbid forgetfulness, and syndrome of to be ill at ease and full of dread.' The effect of $\mathrm{VaD}$ treatment on acupuncture is definite. And the advantage of acupuncture includes simple and convenient, inexpensive, without any toxicity and side effects way for excellent treatment choice. The TCM clinical effect is the most important leading advantage for clinical practice as well as acupuncture. The effect of acupuncture includes dredging meridians and collaterals, strengthening healthy qi to eliminate pathogens, and regulating yin-yang.

Doctrine of meridians and collaterals system has a clear understanding on $\mathrm{VaD}$ while combining with the above standpoints in TCM, especially to be reflected in twelve meridians and collaterals, and eight extra meridians. Acupuncture also has both TCM characteristics of patterns of holistic thought and syndromes differentiation, especially syndromes differentiation of meridians and collaterals. Therapeutic principle of acupuncture includes curing spirit and maintaining $q i$, reinforcing insufficiency and reducing excessiveness, simultaneous treatment of principal and subordinate symptoms, partial and whole treatment. The $\mathrm{VaD}$ treatment with syndromes differentiation in 
acupuncture based on the integration guidance reflects TCM characteristics and objectives of regulating yin-yang. Usually, there are six syndromes of VaD classified for clinical practice in acupoints selection and manipulation. Acupoints should be modification according to syndromes as well as reinforcing and reducing techniques for needling. While two congregated head points ( $\mathrm{Da}$ and xiao Sishencong) are so important to $\mathrm{VaD}$ treatment integrated with our decades of years clinical experience. Besides, ear acupuncture therapy, scalp-acupuncture therapy, hydro-acupuncture therapy, warm needling method, and other acupuncture therapy methods will be also flexible modification to obtain personal medical effect.

\subsection{Syndrome of deficiency of kidney qi and marrow sea}

'The deficiency of marrow sea leads to vertigo and tinnitus, sore waist and weak kneed, delinquency and peacefully sleeping' said in Huangdi's Internal Classics. Deficiency of kidneys leads to deficiency of marrow, both of which cause deficiency of the brain sea. Primary symptoms of syndrome of deficiency of kidney qi and marrow sea contain dizziness, dazzling, cloudiness vision, sore waist and weak kneed, delinquency and peacefully sleeping, daffy and dull expression, dull respondence, decline of memory, morbid forgetfulness, poor judgment ability, disorientation (such as often go wrong direction), discrimination disorder (such as often be unacquainted with familiar people or recognize wrong people), thin and sunken pulse, especially feeble chi pulse, thin and rufous tongue with few lingual fur. The therapeutical principle is to tonify kidneys and nourish marrow, ease mental anxiety and regain consciousness. And the primary acupoints for treatment of the syndrome involve Sishencong (also named as Xiao Sishencong) (EX-HN1), Da Sishencong (Qianding, Houding, Luoque) (DU 21, DU 19, BL 8), Fengfu (DU 16), Dazhu (BL 11), Quchi (LI 11), Hegu (LI 4), Taixi (KI 3), Taichong (LR 3). If dizziness and dazzling were severe, Touwei (ST 8), Shenting (DU 24) and Fengchi (GB 20), would be added. If insomnia and anxiousness were conspicuous, Neiguan and Shenmen would be added. If disorientation and discrimination disorder were severe, Muchuang (GB 16) and Zhengying (GB 17) would be added.

Sishencong is extraordinary point and surrounds Baihui, consisted of four acupoints which locate in DU and BL meridians. The hump of head is yang within yang in TCM, and Baihui which is the house of three yang meridians and five convergent collaterals. Foot taiyang meridian communicates with kidneys. Besides, the relationship of bladder and kidneys is exterior and interior. Especially, DU meridian also communicates both kidneys and heart. Kidneys essence could produce marrow and tonify the brain sea. So the stimulation of Sishencong could tonify kidneys yang $q i$ and regulate diseases derived from the house of cerebral spirit. $\mathrm{Da}$ Sishencong is one of the most effective acupoints and distinguishing features of China national famous veteran teran doctors of TCM of professor Zhangji's experience on $\mathrm{VaD}$ treatment in acupuncture. Da Sishencong surrounds Baihui too. Da Sishencong is also consisted of five acupoints which locate in DU and GB meridians, which could combine Sishencong to enforce the effect of tonifying kidneys and marrow and repairing impaired cognition. Fengfu is convergent point of DU and yang link meridians, which could induce resuscitation. Dazhu is convergent acupoint of meridians of hand and foot taiyang, and one of eight convergent points that said as bone convergence, which could regulate yang $q i$ and resuscitate the cognition. Quchi is the meeting point of five shu points and original point of hand yangming meridian, which is one of strengthening points and 
could free yang $q i$ and eliminate phlegm and promote blood circulation to remove blood stasis. Hegu also is in the meridian of hand yangming, which could dredge the meridian passage and tranquilize and allay excitement. Taixi is the meeting point of five shu points and original point of foot shaoyin meridian, which nourish kidneys yin-yang and remove cardiopyrexia for tranquilization. Taichong is the meeting point of five shu points and original point of foot jueyin meridian, which could calm the liver to stop the wind and disperse stagnated $q i$ and blood of liver. All of the points could cooperate to target at the effective treatment of this $\mathrm{VaD}$ syndrome.

\subsection{Syndrome of liver and kidneys yin deficiency, and the essence and marrow start to fade}

Kidney stores essence and liver stores blood in TCM. The deficiency of essence and blood could lead to feeble marrow sea. Primary symptoms of this syndrome contain dizziness, dazzling, tinnitus and deaf, poor expression of eyes, dumb as a wooden chicken, reticence, memory decline, suspiciousness, visual hallucination, restlessness, sore waist and weak kneed, bizygomatic aestus, insomnia and night sweat, weak and thin body, squamous and dry skin, thin, wiry and frequent pulse, red tongue with few lingual fur. The therapeutical principle of which is to nourish liver and kidneys, strengthening marrow and spirit. And the primary acupoints for treatment of the syndrome involve Xiao Sishencong, Da Sishencong, Touwei, Jingming (BL 1), Fengchi, Fengfu, Neiguan, Shenmen, Quchi, Zulinqi (GB 41). If dizziness and dazzling were severe, Xuanlu (GB 5), Xuanli (GB 6), Jingbailao (EX-HN 15), would be added. If limbs tic occurred, Baxie (EX-UE 9), Bafeng (EX-LE 10), Hegu and Taichong would be added. If insomnia was conspicuous, Anmian (EX-HN 22), Neiguan, Yinxi (HT 6), Taixi, would be added. If fever with yin asthenia was severe, Dazhui (DU 14), Dingchuan (EX-B 1), Fuliu (KT 7), Hegu, would be added.

Touwei is convergent point of meridians of foot shaoyang, foot yangming and yang link, which refresh mental and eyes. Jingming is convergent point of meridians of hand and foot taiyang, foot yangming, yin and yang heels, which could reduce fever and improve acuity of vision, expel wind and promote the restoration of consciousness. Fengchi is convergent point of meridians of hand and foot shaoyang, and yang link, which could calm the liver to stop the wind, expel wind and promote the restoration of consciousness. Neiguan is collateral point of meridian of hand jueyin, and one of eight convergent points to communicate with yin link meridian, which could quiet down the mind and heart, and regulate $q i$ to alleviate mental depression. Shenmen is the transport point of five shu points and original point of meridian of hand shaoyin, which could tranquilize mind and dredge the meridian passage. Zulinqi is the meeting point of five shu points and lower meeting point of meridian of foot yangming, which could invigorate spleen and stomach, strengthen healthy $q i$ to eliminate pathogens, dredge the meridian passage. And all of these points could lead to regulate this $\mathrm{VaD}$ syndrome well.

\subsection{Syndrome of asthenia of both spleen and kidneys, and damage of essence and marrow depletion}

Spleen transports the nutritious substance in the stomach, while kidneys generate marrow and dominate bone in TCM. Asthenia of both spleen and kidneys would lead to marrow sea deficiency. Spleen is as yin zang-organs, so the symptoms of which are special at characteristics of quiet and autistic type. Thus, the primary symptoms of the syndrome 
contain dull expression, reticence, decline of reaction ability, hypomnesis, agnosia, aprophoria, lapsus of reasoning and judgment, weak and thin body, muscular dystrophy, anorexia, short of breath, listless talk, thin, sunken and feeble pulse, light and plump ongue with teeth-marked. The therapeutical principle of which is to strengthen spleen and tonify kidneys. And the primary acupoints for treatment of the syndrome involve Xiao Sishencong, Da Sishencong, Shenting, Fengfu, Dazhui, Quchi, Hegu, Yingu (KI 10), Sanyinjiao (SP 6), Taixi. If insomnia was conspicuous, Neiguan and Shenmen would be added. If yang deficiency was conspicuous, Guanyuan (RN 4) and Qihai (RN 6) would be added. If yin deficiency was conspicuous, Fuliu and Taichong would be added. If the disorders of judgment and orientation were severe, Shenmen, Daling (PC 7), Shenshu (BL 23) would be added.

Shenting is convergent point of meridians of foot taiyang and yangming, and DU, which could quiet down the mind and activate brain, promote flow of yang qi. Dazhui is convergent point of three yang meridians, and DU, which could supplement qi and strengthen yang. Ying $u$ is the meeting point of five shu points of meridian of foot shaoyin, which could nourish kidneys and regulate meridians, and regulate $q i$ to dissipate blood stasis. Sanyinjiao is convergent point of meridians of foot three yin, which could invigorate spleen and stomach, nourish liver and kidneys, and dredge the meridian passage. All of the points, especially leaded with Xiao Sishencong and Da Sishencong, could mend impaired cognition of the syndrome.

\subsection{Syndrome of seven emotions stagnation and hyperactivity of fire of heart and liver}

Fire syndrome is caused by overreaction of the five emotions, such as flaming up of liver fire due to mental irritation. Both heart and liver are yang zang-organs, and the heart controls mental and emotional activities as liver controls dispersion, so hysteria often dues to fire of heart and liver. Primary symptoms of the syndrome involve nervous expression, polylogia, divagation, crankous behavior, visual hallucination, acouasm, restlessness, dizziness, dazzling, conjunctival congestion, constipated, oliguria with reddish urine, wiry, slippery and rapid pulse, red tongue with yellowy fur. The therapeutical principle of which is to clear liver fire and tranquilize by nourishing the heart. And the primary acupoints for treatment of the syndrome involve Xiao Sishencong, Da Sishencong, Dazhui, Dingchuan, Quchi, Hegu, Anmian (EX-HN 22), Zhongwan (RN 12), Liangmen (ST 21), Fenglong (ST 40), Sanyinjiao. If dizziness and dazzling was conspicuous as well as dry throat with bitter taste, Touwei, Toulinqi (GB 15), Taiyang (EX-HN 5), Yingu, would be added. If insomnia was conspicuous, Neiguan, Shenmen, Daling, would be added. If polylogia and paraphasia was conspicuous as well as restlessness, Shenting, Shendao (DU 11), Xingjian (LR 2), Shuigou (DU 26), Fenglong (ST 40), would be added.

Anmian is one of extraordinary points that could quiet down the mind and relieve mental train and strengthen kidneys. Zhongwan is convergent point of meridians of hand taiyang and shaoyang, foot yangming, and RN, which could invigorate spleen and stomach, calm the adverse-rising energy and disperse stagnated liver qi. Liangmen is at the meridian of foot yangming that could regulate stomach to smooth qi, invigorate spleen and soothing liver. Fenglong is collateral point of meridian of foot yangming that could invigorate spleen to remove phlem, regulate stomach to smooth $q i$, reduce phlegm by resuscitation. Therefore, the key effect of this syndrome is to regulate five emotions for ameliorating $\mathrm{VaD}$. 


\subsection{Syndrome of damp abundance due to splenic asthenia and stagnation of phlegm in the orifices}

For qi deficiency of spleen and stomach, transformation failure of spleen and stagnation of phlegm and damp turn out, which lead to turbid phlegm block the orifice of the heart and clear orifices, then, cognition is impaired. Primary symptoms of the syndrome contain dull expression, dementia, emotional instability, mussitation, lassitude and disorders of excessive sleepiness, anorexia, abdominal distention and pain, or oppressive and distending feeling due to stagnation of $q i$, much spittle, heaviness of head, slippery and moisten pulse, light tongue, white and greasy fur on tongue. The therapeutical principle of which is to invigorate spleen to remove phlem and eliminate phlegm for clearing orifices. And the primary acupoints for treatment of the syndrome involve Xiao Sishencong, Da Sishencong, Shenting, Fengfu, Shuigou, Hegu, Fengchi, Zusanli, Fenglong. If insomnia was conspicuous, Neiguan, Shenmen, Daling and Anmian would be added. If anorexia and abdominal distention were conspicuous, Zhongwan, Liangqiu, Tianshu, Taixi, Shenmai, Zhaohai and Yangjiao would be added. If depression was severe, Sanyinjiao, Yanglingquan (GB 34) and Xuanzhong (GB 39) would be added.

Shuigou is the meeting point of meridians of hand and foot yangming, and DU, which could reduce phlegm by resuscitation, and resuscitate by cooling, calm endopathic wind and remove heat. The effect of invigorating spleen to remove phlem and resuscitation with other acupoints show a well match. The integrated with Xiao Sishencong, Da Sishencong, Shenting, Fengfu, Hegu, Fengchi, Zusanli, Fenglong, would target to this VaD syndrome exactly.

\subsection{Syndrome of stagnation of qi to block orifices and blood stagnation in the brain}

For lacking free flow of $q i$ and blood as aging, or rich and fatty diet, which lead to blood stasis, in brain especially that would occur stroke, then, impaired cognition and dementia in the early stage. Primary symptoms of the syndrome contain dull expression, dementia, abnormal thought, eccentric behavior, personality and behaviour disorder, stillness in daytime and dysphoria at night, limbs paralysis, squamous and dry skin, dark eyes, thin and unsmooth pulse, light, purplish and dark tongue with petechiae, thin lingual fur. The therapeutical principle of which is to promote blood circulation to remove blood stasis and induce resuscitation. And the primary acupoints for treatment of the syndrome involve Xiao Sishencong, Da Sishencong, Fengfu, Fengchi, Dazhu, Geshu (BL 17), Xuehai (SP 10), Kongzui (LU 6). If recognition disorder was severe, Shenting and Xinmen (DU 22) would be added. If stroke and disorder of consciousness were severe, Shuigou, Daling, Zhaohai would be added. If deadlimb was conspicuous, Baxie, Bafeng, Neiguan and Fuliu would be added.

Geshu is one of eight convergent points said as blood convergence that could regulate $q i$ to alleviate mental depression, and promote blood circulation to remove meridian obstruction. Xuehai is at the meridian of foot taiyin that could regulate qi and blood, and invigorate spleen to eliminate dampness. Kongzui is at the meridian of hand taiyin that could clear away heat for hemostasis, moisten lungs and regulate qi. All of the points are match with syndrome differentiation and obtain good effect.

\section{Treatment of $\mathrm{VaD}$ in kinds of acupuncture therapy methods}

\subsection{Body acupuncture}

The body acupuncture is the basic method to regulate $\mathrm{VaD}$ with the above syndromes differentiation. And kinds of needle insertion manipulations will be use based on the 
selected acupoints. For examples, horizontal needling will be use for Xiao Sishencong, Da Sishencong, Xinmen, et al. Oblique needling will be use for Shenting, Touwei, et al. Penetration needling will be use for Taixi, Yanlingquan, Neiguan, et al. Deep needling will be use for Sanyinjiao, Zusanli, Fenglong, et al. Shallow needling will be use for Jingming, Yamen, et al. Yet, after obtaining acu-esthesia, manipulating needle to promote acu-esthesia will be enhanced, including manipulations of mild reinforcing and attenuating, reinforcing, reduction, et al.

\subsection{Electroacupuncture}

The electroacupuncture could strengthen acu-esthesia for $\mathrm{VaD}$ treatment. The selected acupoints for electroacupuncture are based on the differentiated syndrome. For examples, syndromes of deficiency of kidney qi and marrow sea of $\mathrm{VaD}$ that Taixi, Dazhu, Hegu will be use. Syndromes of damp abundance due to splenic asthenia and stagnation of phlegm in the orifices of VaD that Xiao Sishencong, Zusanli, Fenglong will be use. Quantity of stimulus electroacupuncture should comply with personal medicine principle.

\subsection{Ears acupuncture}

Ears contain the information of the whole organism in TCM and acupuncture theory. Therefore, ears acupoints are special in the field of acupuncture treatment as well as VaD. The clinical use of ears acupoints methods in china include massotherapy, magnetotherapy, electroacupuncture, blood-letting therapy, needle-embedding therapy, moulded therapy, plaster therapy, injection therapy, et al.

\subsection{Semiconductor laser irradiation therapeutics}

The use of semiconductor laser irradiation therapeutics for $\mathrm{VaD}$ also is based on syndromes differentiation and body acupuncture. After needle insertion manipulations of body acupuncture, some important acupoints will be integrated with low power or middle power semiconductor laser irradiation therapeutics for enhancing the acu-esthesia.

\subsection{Scalp acu-therapy}

Scalp acupuncture could be only use for $\mathrm{VaD}$ treatment as well as integrated with body acupuncture, such as Xiao Sishencong, Da Sishencong. Scalp also contains the information of the whole organism in TCM and acupuncture theory as well as ears. Scalp acupoints are special for $\mathrm{VaD}$ regulation, not only because of the head are both the house of points and disease location, but also the acupuncture theory and therapy methods are integrated to excellent effect. The manipulations include percussopunctator, horizontal needling, cauterized needling therapy, et al.

\subsection{Others}

Others acupuncture therapy methods contain acupoints injection, tongue acupuncture, catgut implantation at acupoint, eyes acupuncture, integrated with moxibustion also called warmed acupuncture, TCM herbals, western medicine, et al. So kinds of acupuncture therapy methods could be integrated for $\mathrm{VaD}$ treatment effectively in China, especially are based on TCM clinical pattern and personality medicine. 


\section{Clinical cases of VaD}

A 65-year-old man was referred for morbid forgetfulness, dull expression and oligologia for 3 months. After nearly 5 decades of years of smoking, he had quit smoking 3 years. The skull examination in mgnetic resonance imaging (MRI) indicated partial branches of mesencephal vascularities had minimal lesion disease without stroke at local hospital 3 months ago. Although he was summated citicoline sodium and other trophic nerve drugs, the symptoms had been advanced still. So acupuncture was required for regulation by the patient's family. The patient has no other risk factors for heart diseases, such as hypertension, hyperlipoidemia, hyperglycaemia. The patient had dull expression, few speech, tied tongue, slurred speech, poor reactive ability, action retardation, disorder of reasoning and judgment, morbid forgetfulness and insomnia, agnosia, acalculia, sore waist and weak knees, muscular dystrophy of limbs, anorexia, short of breath and fatigue, thin, sunken and feeble pulse, light tongue, plump body of tongue with marked teeth, yellowy lingual fur. MRI was used to confirm the diagnosis of $\mathrm{VaD}$ as well as the use of mini-mental state examination (MMSE). While integrated holistic syndrome differentiation pattern to definite the syndrome of asthenia of both spleen and kidneys, and damage of essence and marrow depletion, and the therapeutical principle of which is to strengthen spleen and tonify kidneys. The primary acupoints for treatment of the syndrome involve Xiao Sishencong, Da Sishencong, Shenting, Fengfu, Dazhui, Quchi, Hegu, Yingu, Sanyinjiao, Taixi, Qihai, Shenmen. The use of mild reinforcing and attenuating way to stimulate the acupoints in 1 to 1.5 inch needle maintain half an hour every time, manipulate needles to promote acuesthesia 2 to 3 times, integrated with scalp acupuncture, electroacupuncture and ears acupuncture therapy, and once-daily therapy for strengthening effect. For half years treatment, the patient's impaired cognition obtained an effective improvement, especially in communication and life of quality. The acupoints and manipulations were changed as the change of patient's VaD situation development and prognosis. After one year's treatment in acupuncture with strengthening TCM daily regulation and rehabilitation, the patient's VaD is cure, and the skull MRI indicated well that matching with this aged.

\section{Conclusions}

The understanding of $\mathrm{VaD}$ in TCM and treatment of that in acupuncture are advantaged on clinical effect which also is as the most leading important topic for acupuncture study. Etiopathogenesises of $\mathrm{VaD}$ contain five highlights in TCM, including ageing and asthenia of healthy energy accompanying with concurrent diseases of zang-organs and fu-organs, autolesion of zang-organs due to improper seven emotions, injured by five viscera excessive emotional activities and incoordination of seven emotions, turbid phlegm blocks the clear orifices and clear yang fails to distribution, blood stasis obstructs the collaterals and deficiency of marrow-reservoir. The five primary points on understanding VaD in TCM are integrated with western medicine to guide for clinical practice. There are six syndromes of $\mathrm{VaD}$ classified for clinical practice in acupoints selection and manipulation, including syndromes of deficiency of kidney qi and marrow sea, liver and kidneys yin deficiency and the essence and marrow start to fade, asthenia of both spleen and kidneys and damage of essence and marrow depletion, damp abundance due to splenic asthenia and stagnation of phlegm in the orifices, stagnation of qi to block orifices and blood stagnation in the brain. As result that treatment with syndromes differentiation in acupuncture on $\mathrm{VaD}$ is clear and 
easy for clinical practice, especially to enhance the effect of acupuncture, while integrated with kinds of therapy methods of acupuncture. However, modern science and technology and methodology should be induced to acupuncture for reveal and solve the scientific problems so that the development and use of acupuncture will be widespread and popular, such as randomized controlled trail and meta-analysis of best evidence for guidelines (Fernández et al., 2011; Ho et al., 2011). In conclusion, the use of acupuncture to treat VaD just reflects TCM thinking pattern on regulating diseases effectively which are as partial of thousands years sediments evidence on personal clinical medicine.

\section{References}

Dobson, C.B., Wozniak, M.A., Itzhaki, R.F. (2003). Do infectious agents play a role in dementia? Trends Microbiol 11(7):312-317.

Fernández, P.J., Campoy, G., García Santos, J.M., et al. (2011). Is there a specific pattern of attention deficit in mild cognitive impairment with subcortical vascular features? Evidence from the attention network test, Dement Geriatr Cogn Disord 31(4):268-275.

Fotuhi, M., Hachinski, V., Whitehouse, P.J. (2009). Changing perspectives regarding late-life dementia, Nat Rev Neurol 5(12):649-658.

Jellinger, K.A. (2008). Morphologic diagnosis of 'vascular dementia'-a critical update, J Neurol Sci 270(1-2):1-12.

Konno S., Meyer J.S., Terayama Y., et al. (1997). Classification, diagnosis and treatment of vascular dementia, Drugs Aging 11(5):361-373.

Kerrouche, N., Herholz, K., Mielke, R., et al. (2006). ${ }^{18 F D G ~ P E T ~ i n ~ v a s c u l a r ~ d e m e n t i a: ~}$ differentiation from Alzheimer's disease using voxel-based multivariate analysis, $J$ Cereb Blood Flow Metab 26(9):1213-1221.

Matioli, M.N. \& Caramelli, P. (2010). Limitations in differentiating vascular dementia from Alzheimer's disease with brief cognitive tests, Arq Neuropsiquiatr 68(2):185-188.

Pennisi, G., Ferri, R., Cantone, M., et al. (2011). A review of transcranial magnetic stimulation in vascular dementia, Dement Geriatr Cogn Disord 31(1):71-80.

Schuff, N., Matsumoto, S., Kmiecik, J., et al. (2009). Cerebral blood flow in ischemic vascular dementia and Alzheimer's disease, measured by arterial spin-labeling magnetic resonance imaging, Alzheimer's Dement 5(6):454-462.

Selnes, O.A. \& Vinters, H.V. (2006). Vascular cognitive impairment, Nat clin pract neurol 2(10):538-547.

Traynor, B.BJ.\& Singleton, A.B. (2010). Nature versus nurture: death of a dogma, and the road ahead, Neuron 68(2):196-200.

Zglinicki, T.V.V.S., Lorenz, M., Saretzki, G., et al. (2000). Short telomeres in patients with vascular dementia: an indicator of low antioxidative capacity and a possible risk factor? Lab Invest 80(11):1739-1747.

Zhang, j. (2010). Professor Zhangji's clinical experience of treatment with syndrome differentiation on stubborn diseases, People medical publishing house, Beijing, China, pp.359-366.

Ho, Y.S., So, K.F., Chang, R.C. (2011). Drug discovery from Chinese medicine against neurodegeneration in Alzheimer's and vascular dementia, Chin Med 6(1):15. 


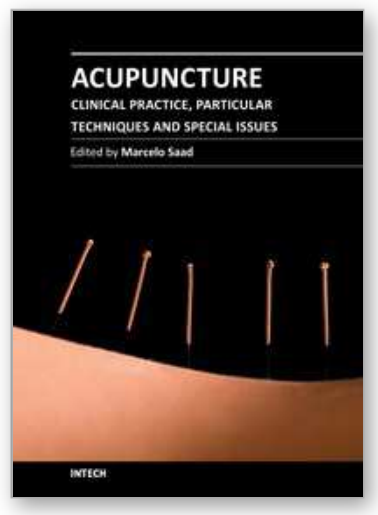

\author{
Acupuncture - Clinical Practice, Particular Techniques and Special \\ Issues \\ Edited by Prof. Marcelo Saad
}

ISBN 978-953-307-630-0

Hard cover, 138 pages

Publisher InTech

Published online 06, September, 2011

Published in print edition September, 2011

Acupuncture is growing in popularity world-wide. Acupuncture and related techniques are useful tools for treating a spectrum of diseases. However, there are still many areas of controversy connected to it due to the fact that mechanisms of action of acupuncture are not entirely clear. Another debilitating element is the absence of a convincing model of sham acupuncture for a control group in clinical trials. Therefore, there are still inappropriate prejudice and unfamiliarity regarding acupuncture. I hope this book can contribute to guide the advance of this ancient medical art. The reader will here find texts wrote by authors from different parts of the world. The chapters cover strategic areas to collaborate with the consolidation of the knowledge in acupuncture. The main objective is to share elements to make acupuncture more and better offered at health systems worldwide.

\title{
How to reference
}

In order to correctly reference this scholarly work, feel free to copy and paste the following:

Liangdeng Zhang, Jie Wang, Kuiwu Yao and Ji Zhang (2011). The Treatment of Vascular Dementia in Acupuncture Based on Syndromes Differentiation, Acupuncture - Clinical Practice, Particular Techniques and Special Issues, Prof. Marcelo Saad (Ed.), ISBN: 978-953-307-630-0, InTech, Available from:

http://www.intechopen.com/books/acupuncture-clinical-practice-particular-techniques-and-special-issues/thetreatment-of-vascular-dementia-in-acupuncture-based-on-syndromes-differentiation

\section{INTECH}

open science | open minds

\section{InTech Europe}

University Campus STeP Ri

Slavka Krautzeka 83/A

51000 Rijeka, Croatia

Phone: +385 (51) 770447

Fax: +385 (51) 686166

www.intechopen.com

\section{InTech China}

Unit 405, Office Block, Hotel Equatorial Shanghai

No.65, Yan An Road (West), Shanghai, 200040, China

中国上海市延安西路65号上海国际贵都大饭店办公楼405单元

Phone: +86-21-62489820

Fax: $+86-21-62489821$ 
(C) 2011 The Author(s). Licensee IntechOpen. This chapter is distributed under the terms of the Creative Commons Attribution-NonCommercialShareAlike-3.0 License, which permits use, distribution and reproduction for non-commercial purposes, provided the original is properly cited and derivative works building on this content are distributed under the same license. 